\title{
Adaptación y validación del cuestionario de motivos de participación para deportistas universitarios colombianos*
}

\author{
Brian Johan Bustos Viviescas** \\ Andrés Alonso Acevedo Mindiola** \\ Jesús Fabián Barrera Pérez \\ Rubén Darío Ramírez Pedraza****
}

Recibido: enero 12 de 2017 • Evaluado: febrero 10 de 2017

Aceptado: febrero 5 de 2016

Resumen

El objetivo del presente estudio fue adaptar y validar el cuestionario de motivos de participación (CMP) para deportistas universitarios colombianos (DUC). Para ello, se realizó un estudio descriptivo-exploratorio con enfoque mixto y una muestra a conveniencia de dieciséis hombres y trece mujeres con una edad comprendida entre los 17 y los 25 años pertenecientes

"Estudio desarrollado en la Universidad de Pamplona, Campus Villa del Rosario durante el periodo abril-junio de 2016, previo al inicio de los Juegos Nacionales ASCUN.

** Estudiante de la Licenciatura en Educación Básica con Énfasis en Educación Física, Recreación y Deportes de la Universidad de Pamplona (Colombia). Correo electrónico: brian. bustos@unipamplona.edu.co

*** Estudiante de la Licenciatura en Educación Básica con Énfasis en Educación Física, Recreación y Deportes de la Universidad de Pamplona (Colombia). Correo electrónico: andres. acevedo@unipamplona.edu.co

${ }^{* * * *}$ Estudiante de la Licenciatura en Educación Básica con Énfasis en Educación Física, Recreación y Deportes de la Universidad de Pamplona (Colombia). Correo electrónico: fabianbarrera_96@hotmail.com

${ }^{* * * * *}$ Estudiante de la Licenciatura en Educación Básica con Énfasis en Educación Física, Recreación y Deportes de la Universidad de Pamplona (Colombia). Correo electrónico: rd.ramirezp@gmail.com 
a los grupos deportivos del Campus Villa del Rosario de la Universidad de Pamplona. Se hizo una adaptación del CMP que constó de 26 ítems. El análisis de la consistencia interna del instrumento y de los ítems ( $\alpha$ de Cronbach), así como las medias y desviaciones típicas, se realizó mediante el paquete estadístico IBM SPSS V.22®. Los resultados obtenidos en el $\alpha$ de Cronbach manifestaron una alta fiabilidad $(\alpha>0,8)$, por lo cual no se eliminó ningún ítem del cuestionario adaptado. En conclusión, la adaptación del CMP presentó una alta fiabilidad en DUC.

Palabras clave: cuestionario, deporte universitario, motivación, validez. 


\section{Adaptation and validation of the questionnaire of reasons for participation for colombian university athletes}

\section{Abstract}

The objective of the present study was adapted and validate the questionnaire of reasons for participation (QRP) for Colombian university athletes (CUA). It conducted a descriptive-exploratory study with blended approach and a sample to convenience of sixteen men and thirteen women aged between 17 and 25 years belonging to the sporting groups of the Campus Villa of the Rosary of the University of Pamplona, an adaptation of the QRP which I had 26 items applied in them. The analysis of the internal consistency of the instrument and the items ( $\alpha$ of Cronbach), as well as the means and standard deviations were carried out in the statistical package IBM SPSS V.22®. The results obtained in $\alpha$ of Cronbach demonstrated high reliability $(\alpha>0.8)$, so any item of the adapted questionnaire was eliminated. In conclusion, the adaptation of the QRP presented a high reliability in CUA.

Keywords: Questionnaire, university sport, motivation, validity 


\section{Introducción}

En materia de salud se han experimentado profundos cambios en los últimos años (Bastías y Stiepovich, 2014), debido a que actualmente la salud es un aspecto en el que se puede influir de manera determinante (Fernández y García, 2010). En vista de ello instituciones en todo el mundo trabajan por la concientización, divulgación y masificación de la actividad física, con el propósito de mejorar la calidad de vida de las personas (Ortega, Palomino, Correa y Montañez, 2013). La actividad física y el deporte se han convertido en un tema de interés para la promoción de la salud en diferentes poblaciones, dado que su práctica regular favorece la disminución en la aparición de diversas enfermedades (Gómez Ruiz, García, Granero y Piéron, 2009), y también es una opción de tratamiento y rehabilitación de estas alteraciones (Rangel, Rojas y Gamboa, 2015). Adicionalmente, dichas prácticas ejercen efectos beneficiosos no solo en el plano físico, sino también en el plano psíquico (Janssen y LeBlanc, 2010; Carrasco y Lepe, 2013).

Estudios realizados en población universitaria colombiana han reportado una baja tasa de actividad física (Gómez, Mateus y Cabrera, 2004; Lema et al., 2009). Este es un indicador alarmante, puesto que señala que los estudiantes universitarios colombianos que no practican actividad física de forma regular pueden llegar a presentar enfermedades crónicas no transmisibles relacionadas con la conducta sedentaria, considerando que hoy en día el sedentarismo es un importante problema de salud pública mundial (Varela, Duarte, Salazar, Lema y Tamayo, 2011), asociado a numerosas patologías como la obesidad, la diabetes, la hipertensión, la arteriosclerosis, la hipercolesterolemia, entre otras (Fernández y García, 2010). Por lo anterior, Farinola y Bazán (2011) destacan la relevancia del estudio en la población universitaria, puesto que existe una baja actividad física y una alta conducta sedentaria.

La efectividad de la actividad física como estrategia de prevención primaria y secundaria de diversos trastornos crónicos ha sido bien documentada (Farinola y Bazán, 2011). Al respecto, García-Laguna, GarcíaSalamanca, Tapiero-Paipa y Ramos (2012) señalan que las universidades buscan promover espacios para sus estudiantes, dedicados a la realización de actividad física y deporte en su tiempo libre, por ejemplo, a través de la vinculación a grupos deportivos institucionales. 
La motivación para la actividad físico-deportiva es una base para estas propuestas educativas (García et al., 2009), por lo tanto, para fomentar una motivación más agradable y conseguir una mayor adherencia a la práctica de la actividad física es fundamental estudiar los perfiles motivacionales (Alías, Aguilar y Hernández-Rodríguez, 2016). Es necesario considerar aspectos de la motivación para la prevención y modificación del sedentarismo, teniendo en cuenta que pocos jóvenes universitarios colombianos realizan actividad física (Varela et al., 2011).

Considerando lo anterior, el estudio de la motivación para la práctica físico-deportiva en universitarios permitirá el análisis de la oferta institucional de los diferentes grupos deportivos, con el fin de animar a los estudiantes para que participen y se mantengan en estos programas que promocionan la vida activa y saludable por medio del deporte. Igualmente, el conocimiento de tales iniciativas será fundamental para organizar y planificar la oferta deportiva de las universidades de Colombia. Sin embargo, los cuestionarios sobre los motivos de participación en deportistas universitarios se han realizado y validado específicamente para la población española, por lo cual no se cuenta con un cuestionario de motivos de participación fiable para la población deportista universitaria colombiana. Por tal razón, el objetivo del presente estudio fue adaptar y validar el cuestionario de motivos de participación en deportistas universitarios colombianos.

\section{Materiales y métodos}

Este es un estudio descriptivo-exploratorio con enfoque mixto y muestra a conveniencia. En este estudio participaron 16 hombres y 13 mujeres con una edad comprendida entre los 17 y los 25 años, estudiantes del Campus Villa de Rosario de la Universidad de Pamplona. A todos se les informó acerca del objetivo del estudio, así como de los instrumentos que se emplearían. Además, se les indicó que los datos recolectados se utilizarían con fines investigativos y que se respetaría la confidencialidad de los mismos.

Los deportistas evaluados pertenecían a los grupos de formación deportiva ofertados por la Universidad de Pamplona, como tenis de campo, rugby, ultimate, voleibol y karate do. Adicionalmente, para poder participar del estudio se tuvieron en cuenta unos criterios de inclusión y exclusión. 
Los criterios de inclusión para participar en el estudio fueron los siguientes:

- Participación voluntaria.

- Estar matriculados en la Universidad de Pamplona y estar inscritos en la actividad/deporte que practicaban.

- Asistencia habitual a las sesiones de entrenamiento del grupo deportivo.

Los criterios de exclusión para no participar en el estudio fueron los siguientes:

- No cumplir los criterios de inclusión.

- Aquellos deportistas que no estuvieron aspirando a participar en Juegos ASCUN, dado que se necesitaban estas características para lograr mayor proximidad a los ajustes realizados en los instrumentos descritos en el siguiente apartado.

\section{Cuestionario de motivos de participación}

Para este estudio, se modificó la versión adaptada de Torres, Carrasco y Medina (2000) para la población universitaria española de la versión en español de Durand (1988) del Participation Motivation Questionnaire, elaborado originalmente por Gill, Gross y Huddleston (1983).

Esta modificación consistió en cambiar el ítem "Quiero mejorar para participar en el trofeo rector" por "Quiero mejorar para participar en los Juegos Universitarios Nacionales ASCUN", dado que este ítem permitía una delimitación más próxima a las motivaciones para la práctica físicodeportiva de los estudiantes universitarios colombianos que participaron en los Juegos ASCUN.

Los datos obtenidos en el cuestionario fueron recolectados y tabulados con el paquete estadístico IBM SPSS V.22®. En este software se realizó el análisis de la consistencia interna del instrumento y de cada ítem por medio del coeficiente $\alpha$ de Cronbach con corrección mediante eliminación de ítem, para ello se tuvieron en cuenta los criterios expuestos por Cicchetti (1988):

- Moderada fiabilidad: $\alpha$ de Cronbach de 0.70 a 0.79.

- Alta fiabilidad: $\alpha$ de Cronbach de 0.80 o valores superiores. 
Así mismo, se calcularon las medidas descriptivas de todos los ítems con media y desviación estándar para poder organizarlos en orden de importancia.

\section{Resultados}

En la tabla 1 se puede observar que en los deportes como tenis de campo, rugby y ultimate hubo mayor participación de los hombres, mientras que en voleibol y karate do participaron más las mujeres.

Tabla 1. Distribución de la muestra por deporte, facultad y sexo

\begin{tabular}{|c|c|c|c|}
\hline \multirow{2}{*}{ Deporte } & \multirow{2}{*}{ Facultad } & \multicolumn{2}{|c|}{ Sexo } \\
\hline & & Hombre & Mujer \\
\hline \multirow{3}{*}{$\begin{array}{l}\text { Tenis de } \\
\text { campo }\end{array}$} & $\begin{array}{l}\text { Ingeniería y } \\
\text { arquitectura }\end{array}$ & 1 & 0 \\
\hline & Educación & 3 & 1 \\
\hline & $\begin{array}{c}\text { Artes y } \\
\text { Humanidades }\end{array}$ & 2 & 0 \\
\hline \multirow{2}{*}{ Rugby } & $\begin{array}{l}\text { Ingeniería y } \\
\text { arquitectura }\end{array}$ & 3 & 0 \\
\hline & Educación & 0 & 1 \\
\hline \multirow{3}{*}{ Ultımate } & $\begin{array}{l}\text { Ingeniería y } \\
\text { arquitectura }\end{array}$ & 2 & 0 \\
\hline & Educación & 1 & 0 \\
\hline & Ciencias Básicas & 4 & 1 \\
\hline
\end{tabular}




\begin{tabular}{cccc}
\hline & $\begin{array}{c}\text { Ingeniería y } \\
\text { arquitectura } \\
\text { Voleibol }\end{array}$ & 2 & 1 \\
& $\begin{array}{c}\text { Artes y } \\
\text { Humanidades }\end{array}$ & 1 & 4 \\
\hline & $\begin{array}{c}\text { Ingeniería y } \\
\text { arquitectura }\end{array}$ & 0 & 1 \\
& $\begin{array}{c}\text { Educación } \\
\text { Artes y } \\
\text { Karate Do }\end{array}$ & 0 & 3 \\
\hline
\end{tabular}

Fuente: elaboración propia.

El coeficiente $\alpha$ de Cronbach obtenido para el cuestionario con todos los ítems fue de 0.84 , por lo cual el cuestionario presenta una alta fiabilidad, como se muestra en la tabla 2.

Tabla 2. Análisis de fiabilidad del instrumento

\begin{tabular}{ccc}
\hline Cuestionario & Número de ítems & $\boldsymbol{\alpha}$ de Cronbach \\
\hline $\begin{array}{c}\text { Cuestionario de motivos de } \\
\text { participación }\end{array}$ & 26 & 0.84 \\
\hline
\end{tabular}

Fuente: elaboración propia.

Los resultados de fiabilidad por medio del coeficiente $\alpha$ de Cronbach de todos los ítems se exponen en la tabla 3. A partir de ello se puede identificar valores similares al obtenido por el instrumento (tabla 2), lo cual representa una alta fiabilidad en todos los ítems, por lo que no se eliminó ninguno de ellos. 
Tabla 3. Análisis de fiabilidad de los ítems del cuestionario

\begin{tabular}{|c|c|}
\hline Ítem & $\alpha$ de Cronbach \\
\hline 1 & 0.83 \\
\hline 2 & 0.83 \\
\hline 3 & 0.84 \\
\hline 4 & 0.85 \\
\hline 5 & 0.83 \\
\hline 6 & 0.84 \\
\hline 7 & 0.83 \\
\hline 8 & 0.84 \\
\hline 9 & 0.84 \\
\hline 10 & 0.84 \\
\hline 11 & 0.83 \\
\hline 12 & 0.84 \\
\hline 13 & 0.83 \\
\hline 14 & 0.83 \\
\hline 15 & 0.84 \\
\hline 16 & 0.84 \\
\hline 17 & 0.82 \\
\hline 18 & 0.84 \\
\hline 19 & 0.84 \\
\hline 20 & 0.82 \\
\hline 21 & 0.83 \\
\hline 22 & 0.85 \\
\hline 23 & 0.83 \\
\hline 24 & 0.84 \\
\hline 25 & 0.83 \\
\hline 26 & 0.82 \\
\hline
\end{tabular}

Fuente: elaboración propia. 
En la tabla 4 se muestran, categorizados por sexo, los valores medios y las desviaciones típicas de cada uno de los 26 ítems analizados en el cuestionario.

Tabla 4. Medias y desviaciones de los motivos contemplados en el cuestionario

\begin{tabular}{lcccc}
\hline & & & & \\
& \multicolumn{2}{c}{ Hombres (19) } & \multicolumn{2}{c}{ Mujeres (13) } \\
& Media & Desv. Est. & Media & Desv. Est. \\
\hline Quiero mejorar mi nivel & 2.79 & 0.54 & 2.92 & 0.28 \\
Me gusta utilizar ropa deportiva & 2.58 & 0.61 & 2.54 & 0.66 \\
Me gusta el deporte en equipo & 2.74 & 0.45 & 2.77 & 0.44 \\
Quiero relajarme & 2.67 & 0.59 & 2.54 & 0.66 \\
Me gusta salir de casa & 2.39 & 0.85 & 2.46 & 0.78 \\
Quiero estar fisicamente bien & 2.94 & 0.24 & 2.92 & 0.28 \\
Me gusta el espíritu de equipo & 2.95 & 0.23 & 2.69 & 0.48 \\
Quiero mantenerme en forma & 2.89 & 0.46 & 2.85 & 0.38 \\
Me siento mucho más sano & 2.74 & 0.56 & 2.77 & 0.44 \\
Me gusta hacer amigos nuevos & 2.53 & 0.70 & 2.15 & 0.69 \\
Me gusta sudar haciendo ejercicio fisico & 2.32 & 0.75 & 2.54 & 0.66 \\
Quiero estar con mis amigos & 2.53 & 0.61 & 2.46 & 0.52 \\
Me gusta hacer ejercicio & 2.58 & 0.69 & 2.62 & 0.65 \\
Me gustan los entrenadores o instructores & 2.37 & 0.68 & 2.46 & 0.52 \\
Me gusta tener algo que hacer & 2.74 & 0.45 & 2.38 & 0.51 \\
Quiero aprender nuevos movimientos o habilidades & 2.84 & 0.37 & 2.62 & 0.77 \\
Tengo cerca la actividad de casa & 2.26 & 0.87 & 2.38 & 0.65 \\
Quiero entretenerme & 2.79 & 0.42 & 2.77 & 0.44 \\
Quiero mejorar mis habilidades & 2.89 & 0.32 & 2.77 & 0.44 \\
Quiero encontrarme estéticamente mejor & 2.37 & 0.76 & 2.38 & 0.77 \\
Me gusta variar de actividad & 2.53 & 0.61 & 2.62 & 0.65 \\
Quiero mejorar para participar en los Juegos Nacionales ASCUN & 2.58 & 0.69 & 2.62 & 0.51 \\
Me gusta pertenecer a un grupo & 2.53 & 0.62 & 2.62 & 0.51 \\
Quiero gastar energí & 2.42 & 0.61 & 2.54 & 0.52 \\
Pretendo perder peso & 1.84 & 0.83 & 2.00 & 0.71 \\
Quiero tener contacto con gente de otras carreras & 2.26 & 0.87 & 2.15 & 0.69 \\
\hline
\end{tabular}

Fuente: elaboración propia.

En la tabla 5 se puede evidenciar que los motivos mejor valorados por los hombres son "Me gusta el espíritu de equipo", "Quiero estar físicamente bien", "Quiero mantenerme en forma", "Quiero mejorar mis habilidades" y "Quiero aprender nuevos movimientos o habilidades", mientras que los motivos menos valorados por los hombres fueron "Pretendo perder peso", "Quiero tener contacto con gente de otras carreras", "Tengo cerca la actividad de casa" y "Me gusta sudar haciendo ejercicio físico". Por ende, las categorías más valoradas fueron forma física-salud y desarrollo de 
habilidades, y las menos valoradas fueron razones situacionales y relaciones amistosas.

Tabla 5. Relevancia de los motivos contemplados en el cuestionario por los hombres

Hombres

(16)

\begin{tabular}{clc} 
\# Ítem & \multicolumn{1}{c}{ Ítem } & Media \\
\hline 25 & Pretendo perder peso & 1.84 \\
26 & Quiero tener contacto con gente de otras carreras & 2.26 \\
17 & Tengo cerca la actividad de casa & 2.26 \\
11 & Me gusta sudar haciendo ejercicio físico & 2.32 \\
20 & Quiero encontrarme estéticamente mejor & 2.37 \\
14 & Me gustan los entrenadores o instructores & 2.37 \\
5 & Me gusta salir de casa & 2.39 \\
24 & Quiero gastar energía & 2.42 \\
23 & Me gusta pertenecer a un grupo & 2.53 \\
21 & Me gusta variar de actividad & 2.53 \\
12 & Quiero estar con mis amigos & 2.53 \\
10 & Me gusta hacer amigos nuevos & 2.53 \\
22 & Quiero mejorar para participar en los Juegos Nacionales ASCUN & 2.58 \\
13 & Me gusta hacer ejercicio & 2.58 \\
2 & Me gusta utilizar ropa deportiva & 2.58 \\
4 & Quiero relajarme & 2.67 \\
3 & Me gusta el deporte en equipo & 2.74 \\
9 & Me siento mucho más sano & 2.74 \\
15 & Me gusta tener algo que hacer & 2.74 \\
18 & Quiero entretenerme & 2.79 \\
1 & Quiero mejorar mi nivel & 2.79 \\
16 & Quiero aprender nuevos movimientos o habilidades & 2.84 \\
19 & Quiero mejorar mis habilidades & 2.89 \\
8 & Quiero mantenerme en forma & 2.89 \\
6 & Quiero estar fisicamente bien & 2.94 \\
7 & Me gusta el espíritu de equipo & 2.95 \\
\hline & &
\end{tabular}

Fuente: elaboración propia.

En la tabla 6 se puede observar que los motivos mejor valorados por las mujeres son "Quiero estar físicamente bien", "Quiero mejorar mi nivel", "Quiero mantenerme en forma", mientras que los motivos menos valorados por las mujeres fueron "Pretendo perder peso", "Quiero tener 
contacto con gente de otras carreras" y "Me gusta hacer nuevos amigos". Por consiguiente, las categorías más valoradas en este grupo fueron forma física-salud y autorrealización, y, las menos valoradas fueron relaciones situacionales y relaciones amistosas.

Tabla 6. Relevancia de los motivos contemplados en el cuestionario por las mujeres

\begin{tabular}{clc}
\hline & & \\
\# Ítem & & $\begin{array}{c}\text { Mujeres } \\
(\mathbf{1 3}) \\
\text { Media }\end{array}$ \\
\hline 25 & Pretendo perder peso & 2.00 \\
26 & Quiero tener contacto con gente de otras carreras & 2.15 \\
10 & Me gusta hacer amigos nuevos & 2.15 \\
15 & Me gusta tener algo que hacer & 2.38 \\
17 & Tengo cerca la actividad de casa & 2.38 \\
20 & Quiero encontrarme estéticamente mejor & 2.38 \\
5 & Me gusta salir de casa & 2.46 \\
12 & Quiero estar con mis amigos & 2.46 \\
14 & Me gustan los entrenadores o instructores & 2.46 \\
4 & Quiero relajarme & 2.54 \\
11 & Me gusta sudar haciendo ejercicio fisico & 2.54 \\
24 & Quiero gastar energía & 2.54 \\
2 & Me gusta utilizar ropa deportiva & 2.54 \\
13 & Me gusta hacer ejercicio & 2.62 \\
16 & Quiero aprender nuevos movimientos o habilidades & 2.62 \\
21 & Me gusta variar de actividad & 2.62 \\
22 & Quiero mejorar para participar en los Juegos Nacionales ASCUN & 2.62 \\
23 & Me gusta pertenecer a un grupo & 2.62 \\
7 & Me gusta el espíritu de equipo & 2.69 \\
3 & Me gusta el deporte en equipo & 2.77 \\
9 & Me siento mucho más sano & 2.77 \\
18 & Quiero entretenerme & 2.77 \\
19 & Quiero mejorar mis habilidades & 2.77 \\
8 & Quiero mantenerme en forma & 2.85 \\
1 & Quiero mejorar mi nivel & 2.92 \\
6 & Quiero estar fisicamente bien & 2.92 \\
\hline & & \\
\hline
\end{tabular}

Fuente: elaboración propia. 


\section{Discusión}

El objetivo del presente estudio fue adaptar y validar el cuestionario de motivos de participación en deportistas universitarios colombianos. A partir de los resultados obtenidos se pudo identificar una alta fiabilidad del instrumento y todos sus ítems para este propósito; igualmente, se pudo evidenciar que el motivo mejor valorado por los estudiantes universitarios que practican actividad físico-deportiva en los grupos deportivos del Campus Villa del Rosario de la Universidad de Pamplona fue la forma física-salud, mientras que las relaciones amistosas y las razones situacionales son aquellas categorías menos valoradas. En este sentido, los participantes que pertenecen a los grupos deportivos de esta entidad lo hacen por motivos principalmente asociados a la salud, además, no tienen mucho interés en relacionarse con los demás, ni tampoco en el clima en el cual se desarrolla la actividad.

Estos mismos resultados se han encontrado en otros estudios, según los cuales la mayoría de los universitarios que practicaban actividades físicodeportivas lo hacían por "salud" (Centro de Investigaciones sociológicas, 1997; Hellín, Moreno y Rodríguez, 2004; Pavón y Moreno, 2006a, 2006b, 2008; Gómez, Ruiz, García, Granero y Pièron, 2009; Fernández y García, 2010; Varela, Duarte, Salazar, Lema y Tamayo, 2011; Cambronero, Eugenia, Chiner y Lucas-Cuevas, 2015). Esta relevancia está posiblemente justificada por el papel de la actividad física y deportiva en la sociedad posmoderna (Gómez et al., 2009).

Por otra parte, entre los hallazgos del estudio realizado por Fernández y García (2010) se identificó que los universitarios gallegos preferían practicar actividad físico-deportiva de forma individual, así como otros estudios mostraron que las relaciones sociales eran uno de los motivos menos valorados por los universitarios (Pavón y Moreno, 2005; Gómez et al., 2009). Mientras que Aguirre y Gonzales (2014) indican que la motivación de los universitarios está orientada por parámetros de regulación extrínseca, en esta investigación la motivación está regulada intrínsecamente (forma física-salud, autorrealización y desarrollo de habilidades).

Por último, se debe recalcar que la actividad físico-deportiva en universitarios se ha convertido en los últimos años en un área de estudio de las ciencias sociales y las ciencias de la actividad física y deporte, porque es en esta etapa de transición a la vida adulta cuando se están consolidando los 
patrones que luego se mantendrán (Valera et al., 2011), con el propósito de generar mayor adherencia en esta población que presenta altos índices de sedentarismo, los cuales pueden traer consigo patologías crónicas no transmisibles como la diabetes, la obesidad, el hipertiroidismo, entre otras.

\section{Conclusión}

Como resultado del estudio, se concluye que la adaptación del cuestionario de motivos de participación presentó una alta fiabilidad en deportistas universitarios colombianos, por lo cual es un instrumento válido para evaluar la motivación para la práctica físico-deportiva en deportistas universitarios del contexto colombiano.

\section{Referencias}

Aguirre Loaiza, H. H. y González Gutiérrez, J. F. (2014). Factores motivacionales, variables deportivas y sociodemográficas en deportistas universitarios. Tesis Psicológica, 9(1), 130-145.

Alías, A., Aguilar, J. M. y Hernández-Rodríguez, A. I. (2016). Motivaciones de los estudiantes universitarios ante la práctica de actividad físico-deportiva de tiempo libre. Las actividades náuticas. Psychology, Society, \& Education, 8(3), 229-242. Recuperado de https://www.psye.org/articulos/Alias.pdf

Bastías Arriagada, E. M. y Stiepovich Bertoni, J. (2014). Una revisión de los estilos de vida de estudiantes universitarios iberoamericanos. Ciencia y enfermería, 20(2), 93-101. doi: https://dx.doi.org/10.4067/S0717-95532014000200010

Cambronero, M., Eugenia Blasco, J., Chiner, E. y Lucas-Cuevas, A. G. (2015). Motivos de participación de los estudiantes universitarios en actividades físicodeportivas. Revista Iberoamericana de Psicología del Ejercicio y el Deporte, 10(2), 179-186.

Carrasco Mantero, M. A. y Lepe Pavón, R. (2013). Motivación de logro y motivación hacia la práctica de la actividad físico-deportiva. Revista electrónica de investigación Docencia Creativa, 2, 163-168. Recuperado de http://hdl.handle. net/10481/27757

Centro de Investigaciones Sociológicas (1997). Informe sobre tiempo libre y deportes. Madrid: CIS. 
Cicchetti, D. V. (1988). Guidelines, criteria, and rules of thumb for evaluating normed and standardized assessment instruments in psychology. Psychological Assessment, 6, 284-290.

Durand, M. (1988). El niño y el deporte. Madrid: Paidós.

Farinolam, M. G. y Bazán, N. E. (2011). Conducta sedentaria y actividad física en estudiantes universitarios: un estudio piloto. Revista argentina de cardiología, 79(4), 351-354.

Fernández, D. A. y García Soidán, J. L. (2010). Motivación hacia la práctica físicodeportiva de universitarios gallegos. Revista de Investigación en Educación, 8, 128-138.

García, M. (coord.) (2009). Dinámicas y estrategias de re-creación.: Más allá de la actividad físico-deportiva. Barcelona, España: Ediciones Grao.

García-Laguna, D. G., García-Salamanca, G. P., Tapiero-Paipa, Y. T. y Ramos C., D. M. (2012). Determinantes de los estilos de vida y su implicación en la salud de jóvenes universitarios. Hacia la Promoción de la Salud, 17(2), 169-185.

Gill D. L., Gross J. B. y Huddleston S. (1983) Participation motivation in youth sports. International Journal of Sport Psychology, 14, 1-14.

Gómez, L. F., Mateus, J. C. y Cabrera, G. (2004). Leisure-time physical activity among women in a neighbourhood in Bogotá, Colombia: Prevalence and socio-demographic correlates. Cadernos de Saúde Pública, 20, 1103-1109.

Gómez López, M., Ruiz Juan, F., García Montes, M. E., Granero Gallegos, A. y Piéron, M. (2009). Motivaciones aludidas por los universitarios que practican actividades físico-deportivas. Revista Latinoamericana de Psicología, 41(3), 519-532.

Hellín, P., Moreno, J. A. y Rodríguez, P. L. (2004). Motivos de práctica físicodeportiva en la Región de Murcia. Cuadernos de Psicología del Deporte. 1 y 2, 4, 101-116.

Janssen, I. y LeBlanc, A. G. (2010). Review systematic review of the health benefits of physical activity and fitness in school-aged children and youth. International Journal of Behavioral Nutrition and Physical Activity, 7, 524 1-16. doi: 10.1186/1479-5868-7-40

Lema, L. F., Salazar, I. C., Varela, M. T., Díaz, J. A., Rubio, A. y Botero, A. (2009). Comportamiento y salud de los jóvenes universitarios: Satisfacción con el estilo de vida. Pensamiento Psicológico, 5(12), 71-88. Recuperado de http://portales. puj.edu.co/psicorevista/components/com_joomlib/ebooks/PS12-5.pdf 
Ortega Parra, A. J., Palomino Gamboa, O. A., Correa, E. y Montañez Acevedo, G. (2013). La Universidad de Pamplona como institución facilitadora y orientadora de la actividad deportiva y recreativa (estudiantes, docentes y administrativos). Revista Actividad Física y Desarrollo Humano, 5(1), 85-91.

Pavón Lores, A. y Moreno, J. A. (2006a). Características de la práctica físico-deportiva en estudiantes universitarios. Revista Conexoes, 4(1), 125-151.

Pavón Lores, A. y Moreno, J. A. (2006b). Diferencias por edad en el análisis de la práctica físico-deportiva de los universitarios. Cuadernos de Psicología del Deporte, 6(1), 53-67.

Pavón Lores, A. y Moreno, J. A. (2008). Actitud de los universitarios ante la práctica físico-deportiva: diferencias por géneros. Revista de Psicología del Deporte, 17(1), 7-23.

Rangel Caballero, L. G., Rojas Sánchez, L. Z. y Gamboa Delgado, E. M. (2015). Sobrepeso y obesidad en estudiantes universitarios colombianos y su asociación con la actividad física. Nutrición Hospitalaria, 31(2), 629-636.

Torres, G., Carrasco, L. y Medina, J. (2000). Investigación sobre los motivos por los que los estudiantes universitarios practican deporte: El caso de la Universidad de Granada. Revista Motricidad, 6, 95-105.

Varela, M. T., Duarte, C., Salazar, I. C., Lema, L. F. y Tamayo, J. A. (2011). Actividad física y sedentarismo en jóvenes universitarios de Colombia: prácticas, motivos y recursos para realizarlas. Colombia Médica, 42(3), 269-277. Recuperado de http://www.bioline.org.br/request?rc11049 